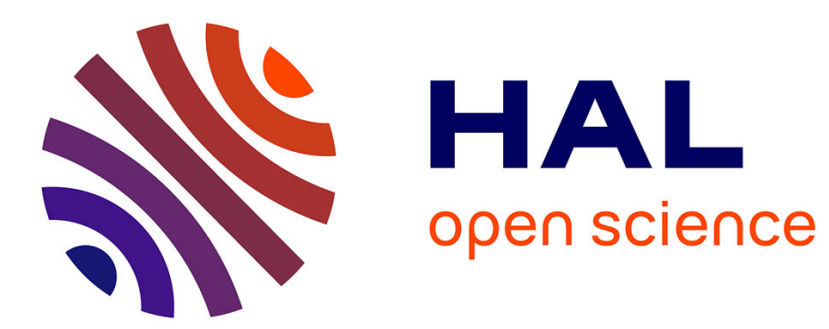

\title{
First results of the NEMO3 experiment
}

\author{
L. Simard
}

\section{To cite this version:}

L. Simard. First results of the NEMO3 experiment. International Europhysics Conference on High Energy Physics (HEP 2003), Jul 2003, Aachen, Germany. pp.S811-S813, 10.1140/epjcd/s2003-03904-7. in2p3-00020433

\section{HAL Id: in2p3-00020433 https://hal.in2p3.fr/in2p3-00020433}

Submitted on 20 Feb 2004

HAL is a multi-disciplinary open access archive for the deposit and dissemination of scientific research documents, whether they are published or not. The documents may come from teaching and research institutions in France or abroad, or from public or private research centers.
L'archive ouverte pluridisciplinaire HAL, est destinée au dépôt et à la diffusion de documents scientifiques de niveau recherche, publiés ou non, émanant des établissements d'enseignement et de recherche français ou étrangers, des laboratoires publics ou privés. 


\title{
First results of the NEMO3 experiment
}

\author{
L. Simard \\ Representing the NEMO Collaboration \\ Laboratoire de l'Accélérateur Linéaire, \\ IN2P3-CNRS et Université de Paris-Sud, Bât. 200, BP 34 - 91898 Orsay Cedex, France
}

\begin{abstract}
The objective of the NEMO Collaboration is to search for neutrinoless double beta decay and thus to investigate physics beyond the Standard Model. The expected sensitivity for the effective neutrino mass is on the order of $0.1 \mathrm{eV}$. The NEMO-3 detector has been operating in the Fréjus Underground Laboratory and has been collecting data since February of 2003. The half-life of two-neutrino double beta decay has been measured for ${ }^{100} \mathrm{Mo}$ and ${ }^{82} \mathrm{Se}$. Constraints on the background for neutrinoless double beta decay have been set.
\end{abstract}

\section{Introduction}

The recent results of neutrino oscillation experiments[1] [2] [3] strongly suggest the existence of massive neutrinos. However these experiments cannot provide the absolute value of the neutrino mass and answer the question of the nature of neutrinos. Are neutrinos Majorana particles, such that neutrinos and antineutrinos are the same particle, or are they Dirac particles, in which case neutrinos and antineutrinos are different? The discovery of neutrinoless double beta decay $(\beta \beta 0 \nu)$ would be a signature that the neutrinos are Majorana particles. Moreover, the observation of neutrinoless double beta decay with experiments like NEMO-3 would constrain the mass spectrum of neutrinos, and their absolute masses.

\section{The NEMO-3 experiment}

\subsection{The NEMO-3 detector}

The main goal of the NEMO-3 experiment[4] is to study neutrinoless double beta decay to a half-life of around $10^{25}$ years. The NEMO-3 detector is located in the Fréjus Underground Laboratory(LSM) under $4800 \mathrm{~m}$ equivalent water to reduce the flux of cosmic rays.

For the search of $\beta \beta 0 \nu$, the isotopes present in the form of foils in NEMO-3 are ${ }^{100} \mathrm{Mo}(6.9$ $\mathrm{kg}),{ }^{82} \mathrm{Se}(0.9 \mathrm{~kg}),{ }^{116} \mathrm{Cd}(0.4 \mathrm{~kg})$, and ${ }^{130} \mathrm{Te}(0.5 \mathrm{~kg})$. Other isotopes, specifically ${ }^{150} \mathrm{Nd}(37 \mathrm{~g})$, ${ }^{96} \mathrm{Zr}(9 \mathrm{~g})$ and ${ }^{48} \mathrm{Ca}(7 \mathrm{~g})$, are used to study two-neutrino double beta decay $(\beta \beta 2 \nu)$. Other sources $(0.6 \mathrm{~kg}$ of natural Tellurium oxide and $0.6 \mathrm{~kg}$ of Copper) are to study the background. 
The thickness of the sources has been optimized to reduce energy loss in the foils. Some of the sources have been purified to reduce the contents of ${ }^{214} \mathrm{Bi}$ and ${ }^{208} \mathrm{Tl}$, which are two isotopes with the large $\mathrm{Q}_{\beta}$-value which contribute to the background for $\beta \beta 0 \nu$.

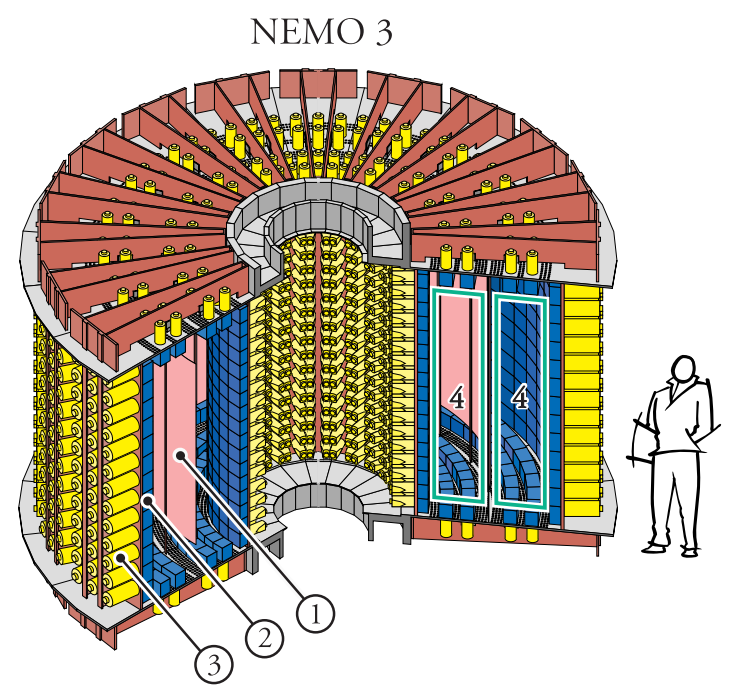

Figure 1: Schematic view of the NEMO-3 detector : (1) source foil, (2) 1940 plastic scintillators coupled to (3) low activity photomultiplier tubes (4) tracking volume with 6180 drift cells operating in Geiger mode.

The particle detection portion of the NEMO-3 detector is $6 \mathrm{~m}$ in diameter and made of two parts. The tracking detector is a wire chamber made of 6180 drift cells in a mixture of helium, $4 \%$ alcohol and 1\% argon and is operating in Geiger mode. The calorimeter consists of 1940 plastic scintillators coupled to low-radioactivity photomultiplier tubes. The typical relative energy RMS for $3 \mathrm{MeV}$ electrons is $\sim 3.5 \%$. The gain stability of the calorimeter is checked regularly with ${ }^{207} \mathrm{Bi}$ calibration source runs and a daily survey of the calorimeter with a laser system[5]. The detector is surrounded by a solenoid, which produces a 25 Gauss magnetic field. Next an $18 \mathrm{~cm}$ thick iron shields for gammas and finally an anti-neutron shielding, made of $35 \mathrm{~cm}$ thick tanks of water and wood. All components of the detector have been tested to have low radioactivity.

The NEMO-3 calorimeter can detect a $1 \mathrm{MeV}$ photon with an efficiency of $50 \%$. The tracking allows one to make a distinction between an electron and a photon and to detect a delayed track, which can correspond to a delayed $\alpha$ desintegration, up to $700 \mu$ s after the first $(\beta, \gamma)$ desintegration. The magnetic field makes it is possible to identify electron from positron.

After a period of test runs in 2002, the detector was completed in the LSM and started to take data in February 2003.

\subsection{The sources of background for $\beta \beta 0 \nu$}

The background can be classified into two types, according to the place where the radioactive decay occurs. Either it can be due to a $(\beta, \gamma)$ desintegration of ${ }^{214} \mathrm{Bi}$ or ${ }^{208} \mathrm{Tl}$ in the source foil. A second electron can be produced either by the photon via the Compton effect, or by the electron via the Möller effect or be a conversion electron [6]. Then the desintegration can mimic a $\beta \beta$ event. 
Photons produced either by radioactive desintegration outside the source foils or by radiative capture in copper or iron of thermal neutrons[7] can produce two high-energy electrons through a double Compton or a Compton followed by a Möller process.

\section{Performances of the detector}

The performance of the detector has been checked with test runs. The reconstruction of verticies was tested with calibration runs involving $60{ }^{207} \mathrm{Bi}$ sources. The ${ }^{207} \mathrm{Bi}$ sour- ces can emit 1 electron. The transverse and longitudinal resolutions obtained (table 1) are in agreement with the specifications[8].

\begin{tabular}{lll}
\hline $\mathrm{e}^{-}$energy & $0.5 \mathrm{MeV}$ & $1 \mathrm{MeV}$ \\
\hline transverse resolution $(\mathrm{cm})$ & 0.3 & 0.2 \\
longitudinal resolution $(\mathrm{cm})$ & 1.1 & 0.7 \\
\hline
\end{tabular}

Table 1: Resolution on vertex reconstruction, obtained with ${ }^{207} \mathrm{Bi}$ calibration runs

The energy calibration is checked using ${ }^{207} \mathrm{Bi}$ and ${ }^{90} \mathrm{Sr}$ calibration runs. ${ }^{207} \mathrm{Bi}$ sources can produce $0.5 \mathrm{MeV}$ or $1 \mathrm{MeV}$ conversion electrons whereas the ${ }^{90} \mathrm{Sr}$ source can be used to test the end-point of the $\beta^{-}$desintegration, at around $2.3 \mathrm{MeV}$. The typical relative energy RMS found for $1 \mathrm{MeV}$ electron, $6 \%$, is in agreement with expectation.

The performance on timing measurements by the PMTs was tested with ${ }^{60} \mathrm{Co}$ (which emits simultaneously two photons) and ${ }^{207} \mathrm{Bi}$ (which can emit two electrons) sources. The tests were is also in agreement with the design.

\section{First preliminary results of physics}

The runs taken in February and March 2003 have been analyzed. They correspond to $650 \mathrm{~h}$ of data collection.

\subsection{Two-neutrino double beta decay $(\beta \beta 2 \nu)$}

The event selection criterion requires two electron tracks to be associated with active PMTs hits, and that the two tracks originate from the same vertex on the foil. Using time-of-flight criteria, the decay can be confirmed as occurring in the source. To reject $\alpha$ particle, no delayed hits should be present near the vertex.

For the ${ }^{100}$ Mo sources, 13750 events were selected, with a signal-to-background ratio of 40 . This study already corresponds to seven times the number of selected events in the operation of the NEMO-2 detector[9]. 


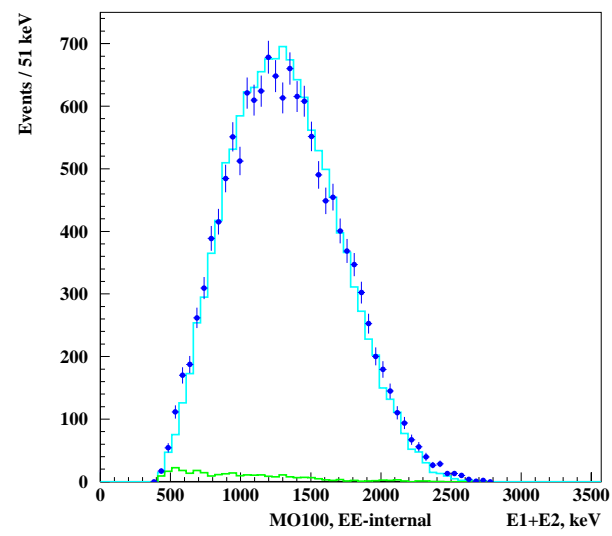

Figure 2: Distribution of the sum of the two electron energies, for ${ }^{100} \mathrm{Mo} \beta \beta 2 \nu$ events in the runs taken during February and March 2003. The crosses represent the data where the background contribution (green line) has been subtracted. The blue line represents the simulation of $2 \beta 2 \nu$ events.

The distribution of the summed energies of the electrons is shown in Fig 2, after substraction of the background. The angular distribution of the two electrons has also been compared with simulations (Fig 3).

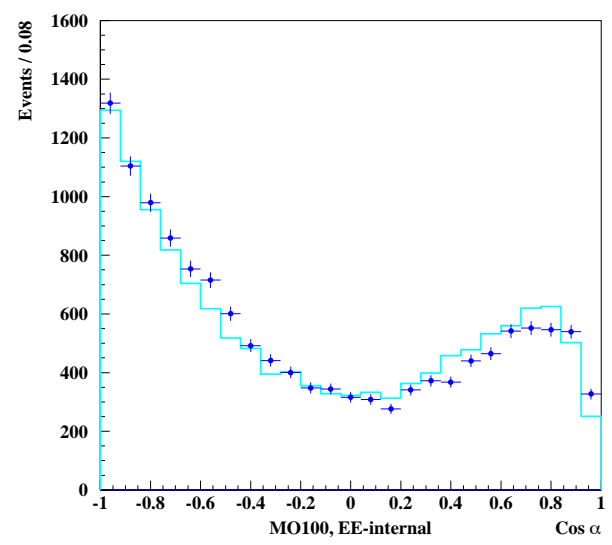

Figure 3: Distribution of the cosine of the angle between the two electrons, for ${ }^{100} \mathrm{Mo} \beta \beta 2 \nu$ events in runs in February and March 2003. The crosses represent the data with the background contribution subtracted. The blue line is the simulated $\beta \beta 2 \nu$ data.

The preliminary value of the $\beta \beta 2 \nu$ half-time decay for ${ }^{100} \mathrm{Mo}$ is :

$$
\mathrm{T}_{1 / 2}=7.8 \pm 0.09 \text { (stat) } \pm 0.8(\text { syst }) 10^{18} \mathrm{y} .
$$

Due to uncertainties in the detector efficiency, a conservative $10 \%$ systematic error has been used. 
The preliminary value of the $\beta \beta 2 \nu$ half-time decay for ${ }^{82} \mathrm{Se}$ is :

$$
\mathrm{T}_{1 / 2}=9.1 \pm 0.4(\text { stat }) \pm 0.9(\text { syst }) 10^{18} \mathrm{y}
$$

\subsection{Study of the background}

The NEMO-3 detector can measure its own background in different channels : one electron with 1, 2, 3 photon channels for ${ }^{208} \mathrm{Tl}$ background and one electron with one alpha particle for the ${ }^{214} \mathrm{Bi}$ background.

\subsection{1 $\quad{ }^{208} \mathrm{Tl}$ background}

To estimate the ${ }^{208} \mathrm{Tl}$ contamination in the source foils, events with one electron and 1,2 or 3 photons were selected. The time-of-flight criteria required the photon(s) emitted from the foil at the same time as the electron. Cuts on the energy of the electron and the photon(s) reduce the contribution of other backgrounds. The limit found for the ${ }^{208} \mathrm{Tl}$ activity in the ${ }^{100} \mathrm{Mo}$ sources is $100 \mu \mathrm{Bq} / \mathrm{kg}$, which is of the same order as the limit obtained with high purity germanium detectors. It will be improved with additional data.

\subsection{2 $\quad{ }^{214} \mathrm{Bi}$ background}

Events initiated with one electron track and followed by one alpha track made of delayed Geiger hits with a common vertex on the foil were culled out(Fig 4). It is the case that most of the ${ }^{214} \mathrm{Bi}$ desintegrations come from the decay of ${ }^{222} \mathrm{Rn}$ which enters the detector, in a very small proportion, from the laboratory air. The typical ${ }^{222} \mathrm{Rn}$ activity is on the order of $30 \mathrm{mBq} / \mathrm{m}^{3}$. This value was confirmed with a radon detector measuring the NEMO-3 gas on its output.

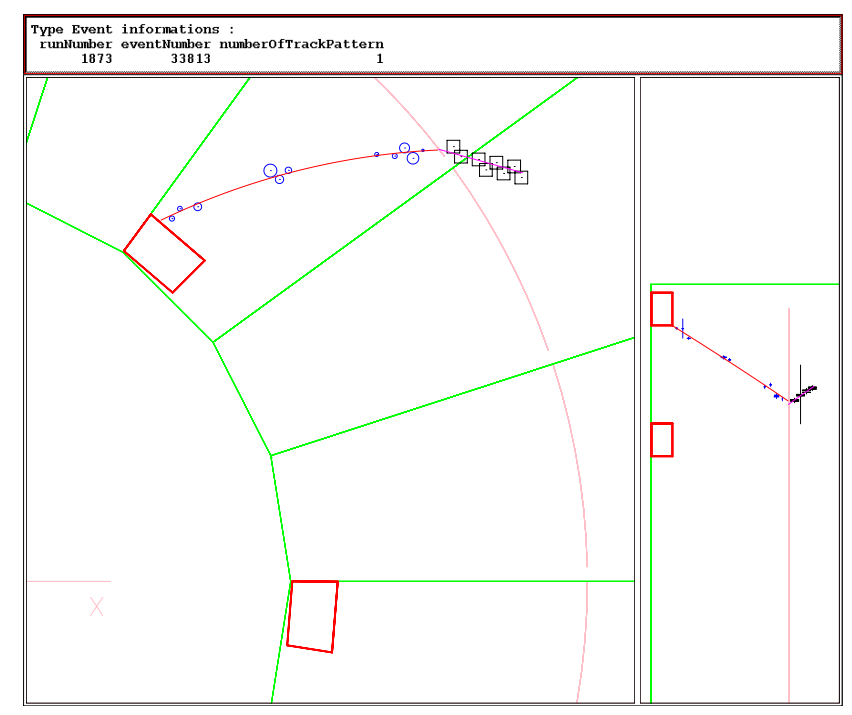

Figure 4: A sample ${ }^{214} \mathrm{Bi}$ event. The circles represent the drift distance to the anodic wire for the triggered Geiger cells, the small boxes identify delayed Geiger cells and the parallelograms the scintillators. The curve connecting the circle is the reconstructed electron track and the short line the reconstructed alpha track. 
This radon level produces on the order of $1 \beta \beta 0 \nu$-like event in per year, which is on the same order of magnitude as for other backgrounds. To reduce the radon level by up to two orders of magnitude, an anti-radon tent surrounding the detector will be built in Fall of 2003 and an radon free air factory will be installed in LSM in Summer of 2004.

\section{Conclusion}

The detector performance has been studied with test runs taken between June and December 2002. The performances of the tracking and calorimeter are in agreement with the design.

Since February 2003, the detector has been running and taking data. Nearly $1700 \mathrm{~h}$ of data were collected by the end of May 2003. For $890 \mathrm{~h}$ of analyzed data, the statistical error on the

half-time decay of ${ }^{100} \mathrm{Mo}$ and of ${ }^{82} \mathrm{Se}$ reach $1 \%$ and $4 \%$. Results for the $\beta \beta 0 \nu$ should come in 2004.

\section{Acknowledgements}

The authors would like to thank the Fréjus Underground Laboratory staff for their technical assistance in building and running the experiment. Part of this work was carried out with the support of Grant INTAS No. 00-00362.

\section{References}

[1] Y. Fukada et al., (SuperKamiokande Collaboration), Phys. Rev. Lett. 81 (1998) 1562.

[2] Q. R. Ahmad et al., (SNO Collaboration), Phys. Rev. Lett. 89 (2001) 11301.

[3] K. Eguchi et al., (KamLand Collaboration), Phys. Rev. Lett. 90 (2003) 21802.

[4] NEMO Collaboration, Preprint LAL 94-29, LAL Orsay, 1994

[5] C. Jollet, PhD thesis, Université Bordeaux-I, 2002.

[6] R. Arnold et al., Nucl. Instr. Meth., A503 (2003) 649.

[7] C. Marquet et al., Nucl. Instr. Meth., A457 (2000) 487.

[8] A.I. Etienvre, PhD thesis, Université Paris-Sud, 2003.

[9] D. Dassié et al., Phys. Rev., D51 (1995) 2090. 